\title{
Star-forming knots and density wave in NGC $2997^{\star}$
}

\author{
P. Grosb $\varnothing 1^{1}$, H. Dottori ${ }^{2}$, and R. Gredel ${ }^{3}$ \\ ${ }^{1}$ European Southern Observatory, Karl-Schwarzschild-Str. 2, 85748 Garching, Germany \\ e-mail: pgrosbol@eso.org \\ 2 Instituto de Física, Univ. Federal do Rio Grande do Sul, Av. Bento Gonçalves 9500, 91501-970 Porto Alegre, RS, Brazil \\ 3 Max-Planck-Institut für Astronomie, Königstuhl 17, 69117 Heidelberg, Germany
}

Received 18 April 2006 / Accepted 2 May 2006

ABSTRACT

\begin{abstract}
Context. Many grand design spiral galaxies show strings of bright knots along their arms on near-infrared $K$-band images. The alignment of such knots suggests a relation to the spiral pattern and possibly to a large-scale, star-forming front associated with a density wave.

Aims. Bright knots in the southern arm of NGC 2997 were studied to determine their nature and evolutionary state.

Methods. Low resolution near-infrared $K$ - and $J$-band spectra of the knots were observed with ISAAC on the VLT.

Results. Most of the knots show strong $\mathrm{H}_{\mathrm{I}} \mathrm{Br}_{\gamma}$ emission with some also having $\mathrm{He} \mathrm{I}$ and $\mathrm{H}_{2}$ emission. A few knots show indications of ${ }^{12} \mathrm{CO}$ absorption. This suggests that the knots are very young stellar clusters with masses up to $5 \times 10^{4} M_{\odot}$.

Conclusions. The knots azimuthal distance from the $K$-band spiral correlates well with their $\mathrm{Br}_{\gamma}$ strength, indicating that they are located inside the co-rotation of the density wave, which triggered them through a large-scale, star-forming front. These relative azimuthal distances suggest an age spread of more than 1.6 Myr, which is incompatible with standard models for an instantaneous star burst. This indicates a more complex star-formation history, such as several bursts or continuous formation.
\end{abstract}

Key words. galaxies: individual: NGC 2997 - galaxies: spiral - galaxies: star clusters - galaxies: structure - infrared: galaxies techniques: spectroscopic

\section{Introduction}

Studying spiral structure on near-infrared (NIR) $K$-band images of disk galaxies, Grosbøl \& Patsis (1998) noticed that many grand design spirals have bright knots along their arms. The alignment of these knots along the arms suggests that they are related to the spiral structure. Patsis et al. (2001) studied such knots in two galaxies using narrow-band filters in the $K$-band and concluded that the major contribution to their $K$-band flux comes from continuum radiation. Furthermore, their small sizes indicate relatively young dynamic ages, as stellar clusters resolve in $<50$ Myr (Wielen 1977). In many cases, they are embedded in dust lanes and invisible in visual bands.

Emitting objects heavily enshrouded in dust were originally detected in radio and far infrared emission (Wood et al. 1988). Old models tried to explain this emission by assuming that we witness the formation of massive stars. Implicitly, these models supposed that the emitting objects were totally obscured in the NIR ( $J$-, $H$-, and $K$-bands). More recently, it was shown that many of these regions were also detected in NIR. Hanson et al. (2002) have shown that approximately 50\% out of 63 radio-selected UCHII regions are visible in NIR bands. Moreover, Conti \& Blum (2002) have found similar behavior within the large star-forming H II region W49A, in which a bunch of NIR-emitting OB star-cocoons surrounds a cluster of around 30 radio-detected OB stars, totally obscured in NIR. W49A presents characteristics similar to those of extragalactic,

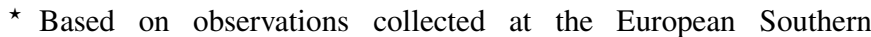
Observatory, Chile; program: ESO 75.B-0579. ultra-dense H II radio sources found in M 33, NGC 253, and NGC 6946 by Johnson et al. (2001).

In this letter, through ISAAC/VLT spectroscopy, we analyze the properties of a series of $K$-band emitting knots detected in the southern arm of NGC 2997, which are neighbors of the H II regions and the associated dust lane, but not exactly coincident with any of them.

\section{Data and reductions}

The southern arm of the grand design spiral galaxy NGC 2997 $\left(\alpha_{2000}=9^{\mathrm{h}} 45^{\mathrm{m}} 38^{\mathrm{s}} .8, \delta_{2000}=-31^{\circ} 11^{\prime} 27^{\prime \prime}\right)$, classified as Sc(s)I.3, displays a long string of well-aligned knots, as can be seen in Fig. 1, which shows a $K$-band image (Grosbøl et al. 2004). Most of the knots are totally embedded in dust lanes and are not visible on $B$-band images (see Fig. 1c). The location of the knots relative to the center of NGC 2997 is given in Table 1, where $K$-band magnitudes are also listed, using an aperture of 1". 4 .

To investigate the nature of these knots, low resolution $K$ and $J$-band spectra were obtained with ISAAC/VLT in service mode on April 28, 2005. The widest ISAAC slit of $2^{\prime \prime}$ was used to include as many knots as possible, with some only partially included. The slit position is shown in Fig. 1b, where the knots are identified. Due to the complex distribution along the slit, target and sky exposures were interleaved in a "TTS" sequence with a sky offset of $1^{\prime}$ to the south. The total on-target exposure times for $K$ - and $J$-band spectra were $30^{\mathrm{m}}$ and $12^{\mathrm{m}}$, respectively. The 6 individual spectra for each band were stacked after they had been sky subtracted, rectified by the standard ESO pipeline, and aligned. The seeing was 0.6 , which gave a spectral resolution 


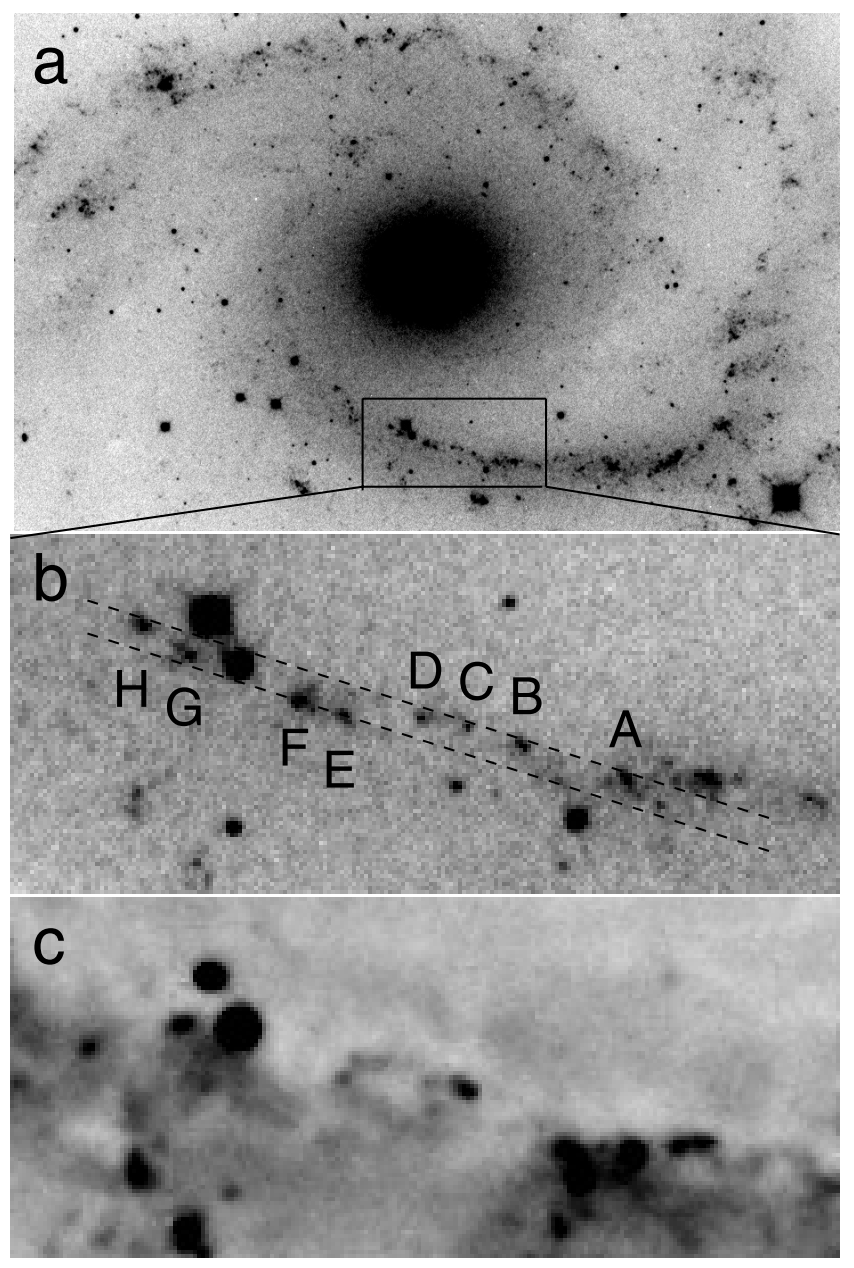

Fig. 1. Direct $K$-band image of NGC 2997: a) inner parts of NGC 2997, b) enlarged part of the $K$ map showing the slit position and identification of knots along it, and c) B map of the same region where the knots are obscured by dust lanes.

of around 750 in $K$ and 860 in $J$ for point sources. Telluric absorption lines in the spectrum of a foreground star located in the slit were used for wavelength calibration. Since the knots were only marginally resolved, the spectra were extracted by fitting a Gaussian profile to their light distribution along the slit, except for knot A, which was clearly extended, where an optimal extraction was applied (Robertson 1986). An early-type telluric standard star was observed the same night under similar, but not identical conditions. Before it was used to correct for telluric absorption, its $\mathrm{HI}$ and $\mathrm{He} \mathrm{I}$ lines were removed manually.

The spectra were corrected for the position of the knots perpendicular to the slit, as measured on the $K$-band acquisition image. Since some knots are only partly in the slit, and spatial distribution of line and continuum emission may differ, this is only a first order correction. Finally, the spectra were rebinned to the rest frame of NGC 2997, using a systemic velocity of $1080 \mathrm{~km} \mathrm{~s}^{-1}$ (Peterson 1978) and noting that the knots are close to the minor axis. The knots $\mathrm{C}$ and $\mathrm{G}$ were not analyzed, as the $\mathrm{S} / \mathrm{N}$ is too low. The remaining $K$-band spectra, having $\mathrm{S} / \mathrm{N}$ in the range of 3-5, are shown in Fig. 3.

\section{Relation to the spiral pattern}

The location of the spiral pattern was determined from the $K$ map (Grosbøl et al. 2004), using the projection parameters

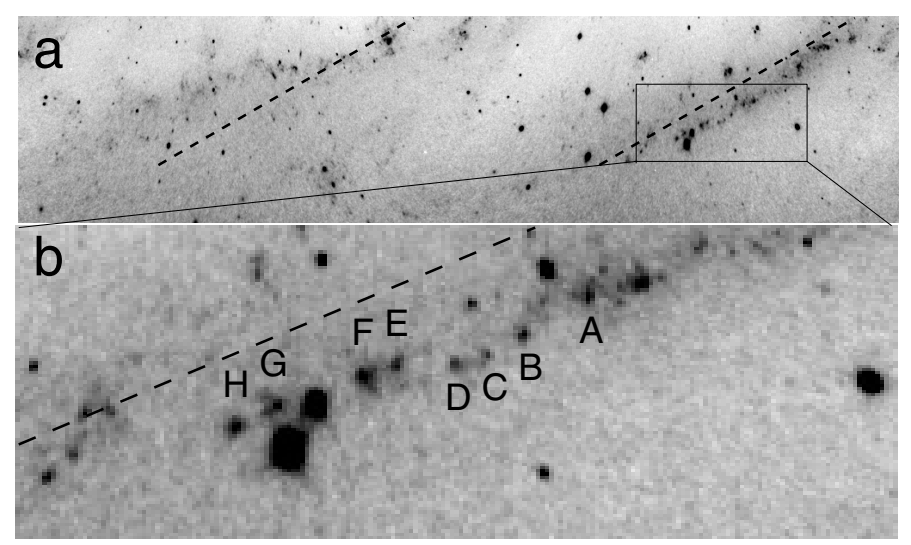

Fig. 2. Polar-logarithmic $\theta-\ln (r)$ map of NGC 2997 in the $K$-band: a) overview map covering $\theta=[0,2 \pi]$ and $r=\left[40^{\prime \prime}, 120^{\prime \prime}\right]$, and b) detail of the map around the knots. The maxima of the azimuthal, two-armed $K$-band intensity variation are shown by dashed lines corresponding to a logarithmic spiral with a pitch angle of $22^{\circ} .9$.

Table 1. Location and magnitude of bright knots observed in NGC 2997. The positions $(\Delta x, \Delta y)$ are relative to the center of NGC 2997, with positive to the East and the North, respectively. The face-on locations $(r, \theta)$ are given relative to the major axis. The azimuthal distance, $\Delta \theta$ between the knots and the two-armed $K$-band intensity maximum, is listed. The absolute magnitude $M_{K}$ is calculated from the apparent magnitude $K^{\prime}$, assuming a distance of $10 \mathrm{Mpc}$.

\begin{tabular}{|c|c|c|c|c|c|c|c|}
\hline Knot & $\Delta x$ & $\Delta y$ & $r$ & $\overline{\theta \theta}$ & $\overline{\Delta \Delta \theta}$ & 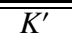 & $\overline{\overline{M_{K}}}$ \\
\hline $\mathrm{A}$ & $-21^{\prime \prime} 4$ & $-58^{\prime \prime} .7$ & $75^{\prime \prime \prime} 2$ & 116.5 & 15.9 & 16.8 & -13.2 \\
\hline B & $-144^{\prime \prime 2}$ & $-56^{\prime \prime} 3$ & $71^{\prime \prime} 0$ & $111: 3$ & 18.4 & 17.2 & -12.8 \\
\hline C & $-10 ! 3$ & $-55^{\prime \prime} 0$ & $68{ }^{\prime \prime} 9$ & $108^{\circ} .4$ & 19.6 & 17.8 & -12.2 \\
\hline D & $-7{ }^{\prime \prime} 6$ & $-54^{\prime \prime} 3$ & $688^{\prime \prime} 0$ & 106.3 & 19.2 & 17.8 & -12.2 \\
\hline E & $-2,2$ & -54.2 & $67 " 99$ & 101.5 & 14.6 & 17.1 & -12.9 \\
\hline $\mathrm{F}$ & $0 .{ }^{\prime} 6$ & $-53^{\prime \prime} .2$ & $67 . .0$ & $99^{\circ} .1$ & $14^{\circ} .1$ & 16.5 & -13.5 \\
\hline G & 8.' 5 & $-50.0^{\prime} 0$ & 64.'1 & 92.2 & $13: 1$ & 17.0 & -13.0 \\
\hline $\mathrm{H}$ & $11^{\prime \prime} 5$ & $-48^{\prime \prime} .0$ & $622^{\prime \prime} 6$ & $89^{\circ} 0$ & 13.2 & 17.1 & -12.9 \\
\hline
\end{tabular}

$(\mathrm{PA}, \mathrm{IA})=\left(102^{\circ}, 38^{\circ}\right)$ by Peterson $(1978)$. A polar-logarithmic map is shown in Fig. 2, where the average linear fit to the twoarmed, azimuthal intensity maxima in the radial range 55-110" is indicated, following the relation $\theta_{2}=0.976+\ln (r) / \tan \left(22^{\circ} .9\right)$. Although the $K$-band pattern is more open inside $75^{\prime \prime}$, the average solution was preferred as better representing the potential. The position of the knots $(r, \theta)$ and their azimuthal distance $\Delta \theta$ from the intensity maximum are listed in Table 1.

The relative azimuthal distances $\Delta \theta$ of the knots correlate well (correlation coefficient of 0.85 ) with their $\mathrm{Br}_{\gamma}$ emission line equivalent width (i.e., age), as listed in Table 2. This suggests that their formation was triggered by a front located more than $20^{\circ}$, in azimuth, from the spiral intensity maximum on the inside, and in general agreement with models of large-scale shocks associated with density waves (Roberts 1969; Yuan \& Grosbøl 1981). The youngest knots, B and D, with strongest $\mathrm{Br}_{\gamma}$ emission, are $4-5^{\circ}$ further away from the spiral arm than the older ones (e.g., knots A, F, and H), which clearly indicates that the knots are located inside the co-rotation of the spiral pattern.

The rotational velocity in the region of the knots is $190 \mathrm{~km} \mathrm{~s}^{-1}$ (Peterson 1978). Assuming that the formation of the knots was triggered by a density wave with a pattern speed $\Omega_{\mathrm{p}}$, azimuthal separations in $\Delta \theta$ can be used to estimate their age differences. A circular rotational model yields a time difference of formation between two knots $\Delta t=\left(\Delta \theta_{1}-\Delta \theta_{2}\right) /\left(\Omega-\Omega_{\mathrm{p}}\right)$. A minimum age difference is obtained for $\Omega_{\mathrm{p}}=0$, while a more 


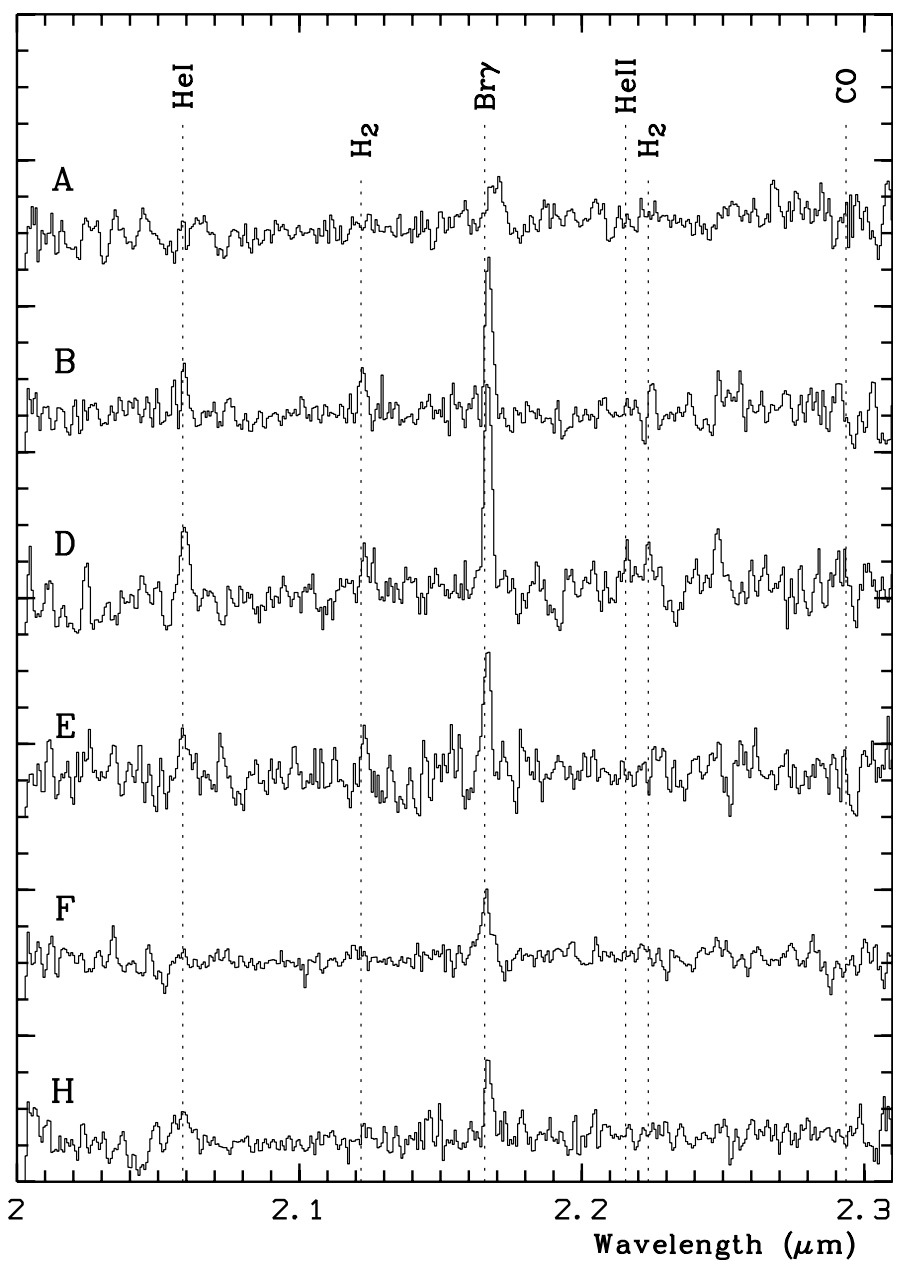

Fig. 3. Low resolution $K$-band spectra of 6 bright knots. They were wavelength calibrated, corrected for telluric absorption, and rebinned to the rest frame of the galaxy. Rest wavelengths of several elements are indicated by dashed lines.

realistic limit is given by $20 \mathrm{~km} \mathrm{~s}^{-1} \mathrm{kpc}^{-1}$ (Grosbøl et al. 1999). For a separation of $6^{\circ}$, this suggests that the minimum age spread must be larger than 1.6 or $2.7 \mathrm{Myr}$, respectively. It is noted that, similarly, $\Omega_{\mathrm{p}}$ can be derived if one can estimate reliable ages for the individual knots from stellar models.

\section{Photometric and spectral properties of knots}

Good examples to compare with NGC 2997 knots are the superstar-clusters $\mathrm{C} 1+\mathrm{C} 2$ (and associated $\mathrm{H}$ II regions $\mathrm{H} 1+\mathrm{H} 2$ ) at the center of NGC 5253 (Alonso-Herrero et al. 2004, hereafter AH04) and W49A (Conti \& Blum 2002), one of the brightest star-forming regions in the Milky Way.

According to Fig. 4 in $\mathrm{AH} 04$, the bulk $\mathrm{C} 1+\mathrm{C} 2 \mathrm{~K}$-band continuum, as well as $\mathrm{Pa}_{\alpha}$ line emission, come from $\mathrm{a} \approx 3^{\prime \prime} \times 1^{\prime \prime} .5$ region, which would appear marginally resolved at the distance of NGC 2997 with our imagery spatial resolution. From AH04 C1 and C2 $M_{F 222 M}$, we deduced $M_{K^{\prime}}(\mathrm{C} 1+\mathrm{C} 2)=-15.3$ and $M_{K^{\prime}}(\mathrm{C} 1)=-13.18$ (assuming $F W H M_{K^{\prime}} / F W H M_{F 222 M}=$ $0.351 / 0.143=2.45$ and constant $K^{\prime}$ continuum), indicating that our six brightest knots are, from this point of view, fully comparable to the $\mathrm{C} 1$ super star cluster. AH04 identify $\mathrm{C} 1$ with an $\mathrm{H}_{\alpha}$ emission peak, or NGC 5253-5 (Turner et al. 2003), while the knots in our sample do not present $\mathrm{H}_{\alpha}$ emission, possibly indicating a higher amount of $A_{\mathrm{V}}$.
Table 2. Equivalent width of main emission lines in the spectra given in $\AA$. Errors are around $10 \%$. Atmospheric emission lines make it difficult to measure $\mathrm{Pa}_{\beta}$ in several cases.

\begin{tabular}{crrrrr}
\hline \hline & $\mathrm{Br}_{\gamma}$ & $\mathrm{Pa}_{\beta}$ & $\mathrm{He}$ & $\mathrm{H}_{2}$ & $\mathrm{H}_{2}$ \\
Knot & $2.166 \mu$ & $1.282 \mu$ & $2.059 \mu$ & $2.123 \mu$ & $2.223 \mu$ \\
\hline $\mathrm{A}$ & 60 & 85 & & & \\
$\mathrm{~B}$ & 115 & & 30 & 18 & 18 \\
$\mathrm{D}$ & 131 & & 61 & 15 & 14 \\
$\mathrm{E}$ & 94 & & 31 & 28 & \\
$\mathrm{~F}$ & 75 & 131 & & & \\
$\mathrm{H}$ & 51 & 60 & 18 & & \\
\hline
\end{tabular}

The $\mathrm{Br}_{\gamma}$ emission line equivalent width is smaller in all the knots than in $\mathrm{C} 1+\mathrm{C} 2(250 \AA)$, knots $\mathrm{D}$ and $\mathrm{B}$ having values higher than $100 \AA$. For bursty and continuous star formation (SF), the equivalent widths of the $\mathrm{H}$ emission lines are anti-correlated with the H II region age (Dottori 1981; AlonsoHerrero et al. 1996). Knot D should be older than $\mathrm{C} 1+\mathrm{C} 2$, but the youngest among the set of NGC 2997 knots. Constraints imposed by kinematics, as discussed in Sect. 3 , indicate that the difference in ages among the youngest and oldest knots should not be lower than 1.6 Myr. Irrespective of the assumed IMF and $M_{\mathrm{u}}$, it is not possible to obtain such age differences from bursty SF inside each one of the knots if the SF is simultaneously triggered along the chain of knots by the density wave in a high metallicity environment, as is that of the knots $(Z \approx 0.02)$. Many specific runs of starburst99, as well as a careful revision of the Leitherer et al. (1999) library, lead us to consider several bursts or a continuous SF with a moderate upper mass limit $M_{\mathrm{u}} \approx 30 M_{\odot}$ as the best solutions for the objects in study. For a canonical Salpeter IMF, we obtain a $\Delta t \approx 2$ Myr. Higher $M_{\mathrm{u}} \approx 100 M_{\odot}$ leads to a very high difference in ages ( $\Delta t \geq 20 \mathrm{Myr})$ among the younger and older knots, neither of which is compatible with the kinematics.

The equivalent width of the emission lines within the $K$-band indicates that they do not influence the $K$-band photometry by more than $10 \%$. Since dust cocoons with temperatures of the order of $30 \mathrm{~K}$ emit mainly at $100 \mu \mathrm{m}$, with a cutoff at $30 \mu \mathrm{m}$, the bulk of the $K$-band continuum may come from non-ionizing stars in their MS or PMS stages, and partially from free-free and free-bound H I emission (Leitherer et al. 1999). Starburst99 furnishes the evolution of $M_{K}$ with time. From Table 1 , for a reddening $A_{\mathrm{V}}=11$ mag similar to that of $\mathrm{C} 1+\mathrm{C} 2$, we infer masses of the order of $5 \times 10^{4} M_{\odot}$ for the knots.

From the theoretical ratio of $\mathrm{Pa}_{\alpha} / \mathrm{Br}_{\gamma}, W\left(\mathrm{~Pa}_{\alpha}\right)$ furnished by AH04 models (their Fig. 6), and our measurement of $W\left(\mathrm{Br}_{\gamma}\right)$, we infer a mass of $\approx 10^{5} M_{\odot}$ for knot $\mathrm{F}$, the brightest of our condensations and $\approx 5 \times 10^{4} M_{\odot}$ for knot D, which is the youngest of the knots, in agreement with the values obtained from the knot's $K$-brightness. The number of $\mathrm{H}$ ionizing photons should be approximately 20 times smaller in $\mathrm{D}$ than in $\mathrm{C} 1+\mathrm{C} 2$. That amounts to $N_{\mathrm{Ly}} \approx 2 \times 10^{51} \mathrm{~s}^{-1}$, or $200 \mathrm{O}$ star "equivalents" (O7V, Vacca 1994), representing two times the flux of Ly photons in W49A (see Conti \& Blum 2002).

Knots A and F clearly show line broadening. Knot A is spatially resolved, so the line broadening might appear because of its extent in the $2^{\prime \prime}$ slit. Knot $\mathrm{F}$ broadening seems to be intrinsic, indicating velocities of the order of hundreds of $\mathrm{km} \mathrm{s}^{-1}$, which might be produced by the shock fronts appearing from the expansion of the cocoon's innermost part, as predicted by canonical models (Churchwell et al. 1990). 
The CO band seems to be present in knots B, D, and E. Since it is a signature of RSG, its presence sets a lower limit of $7 \mathrm{Myr}$ on the age of these knots. CO also constrains $M_{\mathrm{u}}$, since $M_{\mathrm{u}} \approx 100 M_{\odot}$ would lead to $W_{\mathrm{CO}} \approx 12 \AA$, detectable even for our rather low $\mathrm{S} / \mathrm{N}$. The use of $\mathrm{CO}$ as a quantitative constraint would require a higher $\mathrm{S} / \mathrm{N}$.

\section{Conclusion}

A set of $K$-band emitting knots, associated with the southern arm of NGC 2997, with sizes in the order of 50 pc or lower, have been observed spectroscopically in the NIR. The mean absolute $K$ brightness is $M_{K} \approx-13 \mathrm{mag}$, and their present $J$ - and $K$-band emission spectra are similar to cocoon-enshrouded SF regions.

The knots are strikingly aligned along about $4 \mathrm{kpc}$, suggesting a well-synchronized mechanism of piling up the material of which they were formed and for the triggering of star formation that is consistent with a scenario of density wave propagation.

Agreement between large-scale, synchronously triggered SF and knot age differences requires several bursts or continuum SF instead of a single burst within the knots. The youngest of the knots is about $7 \mathrm{Myr}$ old, has a mass of approximately $5 \times$ $10^{4} M_{\odot}$, and needs about $200 \mathrm{O} 7 \mathrm{~V}$ ionizing stars to explain the $\mathrm{Br}_{\gamma}$ line flux. The oldest are about $10 \mathrm{Myr}$ old.

The knots' sizes and masses are similar to that of C1 SSC in NGC 5253, and close to W49A in our galaxy.
Acknowledgements. The ESO ISAAC pipeline and ESO-MIDAS system were used for the reduction and analysis of the data. H.D. thanks a fellowship of the Brazilian Council of Research CNPq, Brazil, and AvH Foundation, Germany.

\section{References}

Alonso-Herrero, A., Aragón-Salamanca, A., Zamorano, J., \& Rego, M. 1996, MNRAS, 278, 417

Alonso-Herrero, A., Takagi, T., Baker, A. J., et al. 2004, ApJ, 612, 222

Churchwell, E., Wolfire, M. G., \& Wood, D. O. S. 1990, ApJ, 354, 247

Conti, P. S., \& Blum, R. D. 2002, ApJ, 564, 827

Dottori, H. A. 1981, Ap\&SS, 80, 267

Grosbøl, P., \& Patsis, P. A. 1998, A\&A, 336, 840

Grosbøl, P. J., Block, David, L., \& Patsis, P. A. 1999, Ap\&SS, 269, 423

Grosbøl, P., Patsis, P. A., \& Pompei, E. 2004, A\&A, 423, 849

Hanson, M. M., Luhman, K. L., \& Rieke, G. H. 2002, ApJS, 138, 35

Johnson, K. E., Kobulnicky, H. A., Massey, P., \& Conti, P. S. 2001, ApJ, 559, 864

Leitherer, C., Schaerer, D., Goldader, J. D., et al. 1999, ApJS, 123, 3

Patsis, P. A., Héraudeau, P., \& Grosbøl, P. 2001, A\&A, 370, 875

Peterson, C. J. 1978, ApJ, 226, 75

Roberts, W. W. 1969, ApJ, 158, 123

Robertson, J. C. 1986, PASP, 98, 1220

Turner, J. L., Beck, S. C., Crosthwaite, L. P., et al. 2003, Nature, 423, 621

Vacca, W. D. 1994, ApJ, 421, 140

Wielen, R. 1977, A\&A, 60, 263

Wood, D. O. S., Churchwell, E., \& Salter, C. J. 1988, ApJ, 325, 694

Yuan, C., \& Grosbøl, P. 1981, ApJ, 243, 432 